\title{
Maximum Matching Width: New Characterizations and a Fast Algorithm for Dominating Set
}

\author{
Jisu Jeong ${ }^{1}$, Sigve Hortemo Sæther ${ }^{2}$, and Jan Arne Telle ${ }^{3}$ \\ 1 Department of Mathematical Sciences, KAIST, 291 Daehak-ro Yuseong-gu \\ Daejeon, South Korea* \\ jjisu@kaist.ac.kr \\ 2,3 Department of Informatics, University of Bergen, Bergen, Norway \\ sigve.sether@ii.uib.no, telle@ii.uib.no
}

\begin{abstract}
We give alternative definitions for maximum matching width, e.g. a graph $G$ has $\operatorname{mmw}(G) \leq k$ if and only if it is a subgraph of a chordal graph $H$ and for every maximal clique $X$ of $H$ there exists $A, B, C \subseteq X$ with $A \cup B \cup C=X$ and $|A|,|B|,|C| \leq k$ such that any subset of $X$ that is a minimal separator of $H$ is a subset of either $A, B$ or $C$. Treewidth and branchwidth have alternative definitions through intersections of subtrees, where treewidth focuses on nodes and branchwidth focuses on edges. We show that mm-width combines both aspects, focusing on nodes and on edges. Based on this we prove that given a graph $G$ and a branch decomposition of mm-width $k$ we can solve Dominating Set in time $O^{*}\left(8^{k}\right)$, thereby beating $O^{*}\left(3^{\operatorname{tw}(G)}\right)$ whenever $\operatorname{tw}(G)>$ $\log _{3} 8 \times k \approx 1.893 k$. Note that $\operatorname{mmw}(G) \leq \operatorname{tw}(G)+1 \leq 3 \mathrm{mmw}(G)$ and these inequalities are tight. Given only the graph $G$ and using the best known algorithms to find decompositions, maximum matching width will be better for solving Dominating Set whenever $\operatorname{tw}(G)>1.549 \times \operatorname{mmw}(G)$.
\end{abstract}

1998 ACM Subject Classification G.2.2 Graph Theory

Keywords and phrases FPT algorithms, treewidth, dominating set

Digital Object Identifier 10.4230/LIPIcs.IPEC.2015.212

\section{Introduction}

The treewidth $\operatorname{tw}(G)$ and branchwidth bw $(G)$ of a graph $G$ are connectivity parameters of importance in algorithm design. By dynamic programming along the associated tree decomposition or branch decomposition one can solve many graph optimization problems in time linear in the graph size and exponential in the parameter. For any graph $G$, its treewidth and branchwidth are related by bw $(G) \leq \operatorname{tw}(G)+1 \leq \frac{3}{2}$ bw $(G)$ [15]. The two parameters are thus equivalent with respect to fixed parameter tractability (FPT), with a problem being FPT parameterized by treewidth if and only if it is FPT parameterized by branchwidth. For some of these problems the best known FPT algorithms are optimal, up to some complexity theoretic assumption. For example, Minimum Dominating Set Problem can be solved in time $O^{*}\left(3^{\operatorname{tw}(G)}\right)$ when given a decomposition of treewidth tw $(G)$ [17] but not in time $O^{*}\left((3-\varepsilon)^{\operatorname{tw}(G)}\right)$ for any $\varepsilon>0$ unless the Strong Exponential Time Hypothesis (SETH) fails $[12]$.

* The first author is supported by Basic Science Research Program through the National Research Foundation of Korea (NRF) funded by the Ministry of Science, ICT \& Future Planning (2011-0011653).

(c) (1) 0 Jisu Jeong, Sigve Hortemo Sæther, and Jan Arne Telle;

cc) licensed under Creative Commons License CC-BY

10th International Symposium on Parameterized and Exact Computation (IPEC 2015).

Editors: Thore Husfeldt and Iyad Kanj; pp. 212-223

Leibniz International Proceedings in Informatics

LI PICS Schloss Dagstuhl - Leibniz-Zentrum für Informatik, Dagstuhl Publishing, Germany 
Recently, a graph parameter equivalent to treewidth and branchwidth was introduced, the maximum matching width (or mm-width) $\mathrm{mmw}(G)$, defined by a branch decomposition over the vertex set of the graph, using the symmetric submodular cut function obtained by taking the size of a maximum matching of the bipartite graph crossing the cut (by König's Theorem equivalent to minimum vertex cover) [18]. For any graph $G$ we have $\operatorname{mmw}(G) \leq \operatorname{bw}(G) \leq \operatorname{tw}(G)+1 \leq 3 \mathrm{mmw}(G)$ and these inequalities are tight, for example any balanced decomposition tree will show that $\operatorname{mmw}\left(K_{n}\right)=\left\lceil\frac{n}{3}\right\rceil$.

In this paper we show that given a branch decomposition over the vertex set of mm-width $k$ we can solve Dominating Set in time $O^{*}\left(8^{k}\right)$. This runtime beats the $O^{*}\left(3^{\mathrm{tw}(G)}\right)$ algorithm for treewidth [17] whenever $\operatorname{tw}(G)>\log _{3} 8 \times k \approx 1.893 k$. If we assume only $G$ as input, then since mm-width has a submodular cut function [16] we can approximate mm-width to within a factor $3 \mathrm{mmw}(G)+1$ in $O^{*}\left(2^{3 \mathrm{mmw}(G)}\right)$ time using the generic algorithm of [13], giving a total runtime for solving dominating set of $O^{*}\left(2^{9 \mathrm{mmw}(G)}\right)$. For treewidth we can in $O^{*}\left(2^{3.7 \mathrm{tw}(G)}\right)$ time [1] get an approximation to within a factor $(3+2 / 3) \operatorname{tw}(G)$ giving a total runtime for solving dominating set of $O^{*}\left(3^{3.666 \mathrm{tw}(G)}\right) .{ }^{1}$ This implies that on input $G$, using maximum matching width gives better exponential factors whenever $\operatorname{tw}(G)>1.549 \mathrm{mmw}(G)$.

Our results are based on a new characterization of graphs of mm-width at most $k$, as intersection graphs of subtrees of a tree. It can be formulated as follows, encompassing analogous formulations for all three parameters mm-width (respectively treewidth, respectively branchwidth):

For any $k \geq 2$ a graph $G$ on vertices $v_{1}, v_{2}, \ldots, v_{n}$ has $\operatorname{mmw}(G) \leq k(\operatorname{resp} \cdot \operatorname{tw}(G) \leq k-1$, resp. bw $(G) \leq k$ ) if and only if there exist a tree $T$ of max degree at most 3 with nontrivial subtrees $T_{1}, T_{2}, \ldots, T_{n}$ such that if $v_{i} v_{j} \in E(G)$ then subtrees $T_{i}$ and $T_{j}$ have at least one node (resp. node, resp. edge) of $T$ in common and for each edge (resp. node, resp. edge) of $T$ there are at most $k$ subtrees using it.

Thus, while treewidth has a focus on nodes and branchwidth a focus on edges, mm-width combines the aspects of both. We also arrive at the following alternative characterization: a graph $G$ has $\operatorname{mmw}(G) \leq k$ if and only if it is a subgraph of a chordal graph $H$ and for every maximal clique $X$ of $H$ there exists $A, B, C \subseteq X$ with $A \cup B \cup C=X$ and $|A|,|B|,|C| \leq k$ such that any subset of $X$ that is a minimal separator of $H$ is a subset of either $A, B$ or $C$. In fact, using techniques introduced by Bodlaender and Kloks [4] these new characterizations will also allow us to compute a branch decomposition of optimal mm-width in FPT time [9]. In Section 2 we give definitions. In Section 3 we define unique minimum vertex covers for any bipartite graph, show some monotonicity properties of these, and use this properties to give the new characterizations of mm-width. In Section 4 we give the dynamic programming algorithm for dominating set. We end in Section 5 with some discussions.

\section{Definitions}

For a simple and loopless graph $G=(V, E)$ and its vertex $v$, let $N(v)$ be the set of all vertices adjacent to $v$ in $G$, and $N[v]=N(v) \cup\{v\}$. For a subset $S$ of $V(G)$, let $N(S)$ be the set of all vertices that are not in $S$ but are adjacent to some vertex of $S$ in $G$, and $N[S]=N(S) \cup S$.

A tree decomposition of a graph $G$ is a pair $\left(T,\left\{X_{t}\right\}_{t \in V(T)}\right)$ consisting of a tree $T$ and a family $\left\{X_{t}\right\}_{t \in V(T)}$ of vertex sets $X_{t} \subseteq V(G)$, called bags, satisfying the following three

\footnotetext{
1 Note that there is also an $O^{*}\left(c^{\mathrm{tw}(G)}\right)$ time 3-approximation of treewidth [3], but the $c$ is so large that the approximation alone has a bigger exponential part than the entire Dominating Set algorithm when using the 3.666-approximation.
} 
conditions:

1. each vertex of $G$ is in at least one bag,

2. for each edge $u v$ of $G$, there exists a bag that contains both $u$ and $v$, and

3. for vertices $u, v, w$ of $T$, if $v$ is on the path from $u$ to $w$, then $X_{u} \cap X_{w} \subseteq X_{v}$.

The width of a tree decomposition $\left(T,\left\{X_{t}\right\}_{t \in V(T)}\right)$ is $\max _{t \in V(T)}\left|X_{t}\right|-1$. The treewidth of $G$, denoted by $\operatorname{tw}(G)$, is the minimum width over all possible tree decompositions of $G$.

A branch decomposition over $X$, for some set of elements $X$, is a pair $(T, \delta)$, where $T$ is a tree over vertices of degree at most 3 , and $\delta$ is a bijection from the leaves of $T$ to the elements in $X$. Any edge $a b$ disconnects $T$ into two subtrees $T_{a}$ and $T_{b}$. Likewise, any edge $a b$ partitions the elements of $X$ into two parts $A$ and $B$, namely the elements mapped by $\delta$ from the leaves of $T_{a}$, and of $T_{b}$, respectively. An edge $a b \in E(T)$ is said to induce the partition $(A, B)$.

A rooted branch decomposition is a branch decomposition $(T, \delta)$ where we subdivide an edge of $T$ and make the new vertex the root $r$. In a rooted branch decomposition, for an internal vertex $v \in V(T)$, we denote by $\delta(v)$ the union of $\delta(l)$ for all leaves of $l$ having $v$ as its ancestor.

Given a symmetric $(f(A)=f(\bar{A}))$ function $f: 2^{X} \rightarrow \mathbb{R}$, using branch decompositions over $X$, we get a nice way of defining width parameters: For a branch decomposition $(T, \delta)$ and edge $e \in T$, we define the $f$-value of the edge $e$ to be the value $f(A)=f(B)$ where $A$ and $B$ are the two parts of the partition induced by $e$ in $(T, \delta)$, denoted $f(e)$. We define the $f$-width of branch decomposition $(T, \delta)$ to be the maximum $f$-value over all edges of $T$, denoted $f(T, \delta): \max _{e \in T}\{f$-value of $e\}$. For set $X$ of elements, we define the $f$-width of $X$ to be the minimum $f$-width over all branch decompositions over $X$. If $|X| \leq 1$, then $X$ admits no branch decomposition and we define its $f$-width to be $f(\emptyset)$.

For a graph $G$ and a subset $S \subseteq E(G)$, the branchwidth bw $(G)$ of $G$ is the $f$-width of $E(G)$ where $f: 2^{E(G)} \rightarrow \mathbb{R}$ is a function such that $f(S)$ is the number of vertices that are incident with an edge in $S$ as well as an edge in $E(G) \backslash S$.

The Maximum Matching-width of a graph $G$, mm-width in short, is a width parameter defined through branch decompositions over $V(G)$ and the cardinality of matchings. For a subset $S \subseteq V(G)$, the Maximum Matching-value is defined to be the size of a maximum matching in $G[S, V(G) \backslash S]$, denoted $\operatorname{mm}(S)$. The mm-width of a graph $G$, denoted mmw $(G)$, is the $f$-width of $V(G)$ for $f=\mathrm{mm}$.

\section{Subtrees of a tree representation for $\mathbf{m m}$-width}

\subsection{König covers}

In this subsection, we will define canonical minimum vertex covers for any bipartite graph. Our starting point is a well-known result in graph theory.

- Theorem 1 (König's Theorem [10]). Given a bipartite graph $G$, for any maximum matching $M$ and minimum vertex cover $C$ of $G$, the number of edges in $M$ is the same as the number of vertices in $C ;|M|=|C|$.

Let $(A, B)$ be the vertex partition of $G$. This statement can be proved in multiple ways. The harder direction, that a maximum matching is never smaller than a minimum vertex cover, does not hold for general graphs, and is usually proven by taking a maximum matching $M$ and constructing a vertex cover $C$ having size exactly $|M|$, as follows:

For each edge $a b \in M$ (where $a \in A$, and $b \in B$ ), if $a b$ is part of an alternating path starting in an unsaturated vertex of $A$, then put $b$ into $C$, otherwise put $a$ into $C$. 

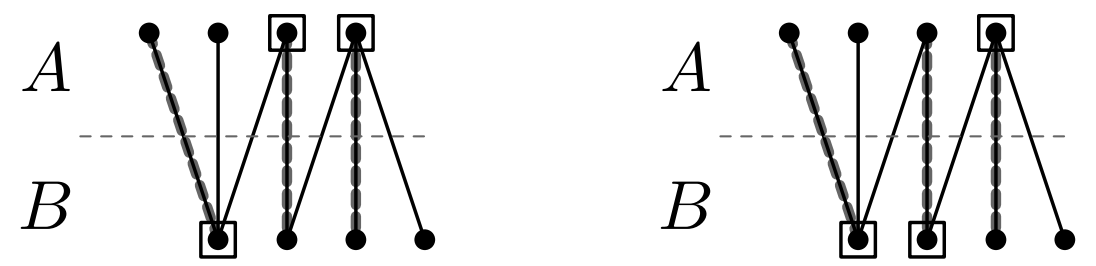

Figure $1 A$-König cover and $B$-König cover.

For a proof that $C$ indeed is a minimum vertex cover of $G$, see e.g. [7]. We will call the vertex cover $C$ constructed by the above procedure the $A$-König cover of $G$. A B-König cover of $G$ is constructed similarly by changing the roles of $A$ and $B$ (see Figure 1).

Lemma 2 below shows that the $A$-König cover will, on the $A$-side consist of the $A$-vertices in the union over all minimum vertex covers, and on the $B$-side will consist of the $B$-vertices in the intersection over all minimum vertex covers.

- Lemma 2. For a bipartite graph $G=(A \cup B, E)$ and minimum vertex cover $C$ of $G$, the set $C$ is the $A$-König cover of $G$ if and only if for any minimum vertex cover $C^{\prime}$ of $G$ we have $A \cap C^{\prime} \subseteq A \cap C$, and $B \cap C^{\prime} \supseteq B \cap C$.

Proof. Let $M$ be a maximum matching of $G$, and $C^{*}$ the $A$-König cover of $G$ constructed from $M$. Since both $C^{*}$ and $C$ are minimum vertex covers, by showing that for any minimum vertex cover $C^{\prime}$ of $G$ we have $A \cap C^{\prime} \subseteq A \cap C^{*}$, and $B \cap C^{\prime} \supseteq B \cap C^{*}$, as a consequence will also show that $C^{\prime}=C^{*}$ if and only if for all minimum vertex covers $C^{\prime}$ of $G$ we have $A \cap C^{\prime} \subseteq A \cap C$ and $B \cap C^{\prime} \supseteq B \cap C$. So this is precisely what we will do.

Let $C^{\prime}$ be any minimum vertex cover, and $b$ any vertex in $C^{*} \cap B$. We will show that $b \in C^{\prime}$, and from that conclude $B \cap C^{\prime} \supseteq B \cap C^{*}$. As $b \in C^{*}$ there must be some alternating path from $b$ to an unsaturated vertex $u \in A$. The vertices $b$ and $u$ are on different sides of the bipartite graph, so the alternating path $P$ between $u$ and $b$ must be of some odd length $2 k+1$. From Theorem 1, we deduce that one and only one endpoint of each edge in $M$ must be in $C^{\prime}$. As each vertex in $V(P)$ is incident with at most two edges of $P$, and all edges of $P$ must be covered by $C^{\prime}$, we need at least $\lceil(2 k+1) / 2\rceil=k+1$ of the vertices in $V(P)$ to be in $C^{\prime}$. However, the vertices of $V(P)-b$ are incident with only $k$ edges of $M$. Therefore at most $k$ of the vertices $V(P)-b$ can be in $C^{\prime}$. In order to have at least $k+1$ vertices from $V(P)$ in $C^{\prime}$ we thus must have $b \in C^{\prime}$.

We now show that $C^{\prime} \cap A \subseteq C^{*} \cap A$ by showing that $a \in C^{*}$ if $a \in A \cap C^{\prime}$. Let $E^{*}$ and $E^{\prime}$ be the edges of $G$ not covered by $C^{*} \cap B$ and $C^{\prime} \cap B$, respectively. Since $C^{*} \cap B \subseteq C^{\prime} \cap B$, the set $E^{*}$ must contain all the edges of $E^{\prime}$. As $C^{\prime}$ is a minimum vertex cover, and all edges other than $E^{\prime}$ are covered by $C^{\prime} \cap B$, a vertex $a$ of $A$ is in $C^{\prime}$ only if it covers an edge $e \in E^{\prime}$. As $E^{\prime} \subseteq E^{*}$, we have $e \in E^{*}$, and hence $C^{*}$ must also cover $e$ by a vertex in $A$. As $G$ is bipartite, the only vertex from $A$ that covers $e$ is $a$, and we can conclude that $a \in C^{*}$.

The following lemma establishes an important monotonicity property for $A$-König covers. For a set $S$ of vertices and a vertex $v$, denote $S+v=S \cup\{v\}$.

- Lemma 3. Given a graph $G$ and tripartition $(A, B, X)$ of the vertices $V(G)$, the following two properties holds for the $A$-König cover $C_{A}$ of $G[A, B \cup X]$ and any minimum vertex cover $C$ of $G[A \cup X, B]$.

1. $A \cap C \subseteq A \cap C_{A}$

2. $B \cap C \supseteq B \cap C_{A}$. 
Proof. To prove this, we will show that it holds for $X=\{x\}$, and then by transitivity of the subset relation and that a König cover is also a minimum vertex cover, it must hold also when $X$ is any subset of $V(G)$.

Let $A^{\prime}=A+x$ and $B^{\prime}=B+x$, and let $C^{\prime}$ be the $A$-König cover of the graph $G[A, B]$ (be aware that this graph has one less vertex than $G$ ). We will break the proof into four parts, namely $A \cap C \subseteq A \cap C^{\prime}, A \cap C^{\prime} \subseteq A \cap C_{A}, B \cap C_{A} \subseteq B \cap C^{\prime}$, and $B \cap C^{\prime} \subseteq B \cap C$. Again, by transitivity of the subset relation, this will be sufficient for our proof. We now look at each part separately.

$A \cap C \subseteq A \cap C^{\prime}$ : Two cases: $|C|=\left|C^{\prime}\right|$ and $|C|>\left|C^{\prime}\right|$. We do the latter first. This means that $C^{\prime} \cup\{x\}$ must be a minimum vertex cover of $G\left[A^{\prime}, B\right]$. Therefore the $A^{\prime}$-König cover $C^{*}$ of $G\left[A^{\prime}, B^{\prime}\right]$ must contain $\left(C^{\prime} \cup\{x\}\right) \cap A^{\prime}$. This means that $C^{*}$ is a minimum vertex cover of $G[A, B]$, and by $C^{\prime}$ being the $A$-König cover of $G[A, B]$, we have from Lemma 2 that $C^{\prime} \cap A \supseteq C^{*} \cap A$. And since $C^{*}$ is a $A^{\prime}$-König cover of $G\left[A^{\prime}, B\right]$ we have $C^{\prime} \cap A^{\prime} \supseteq C \cap A^{\prime}$ and can conclude that $C^{\prime} \cap A \supseteq A \cap C$. Now assume that the two vertex covers are of equal size. Clearly $x \notin C$, as then $C-x$ is a smaller vertex cover of $G[A, B]$ than $C^{\prime}$, so $x$ is not in $C$. This means that $C$ is a minimum vertex cover of $G[A, B]$, so all vertices in $A \cap C$ must be in $C^{\prime}$ by Lemma 2 .

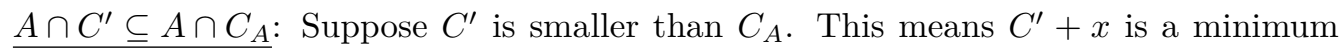
vertex cover of $G\left[A, B^{\prime}\right]$, and hence $\left(C^{\prime}+x\right) \cap A \subseteq C_{A} \cap A$ by Lemma 2. On the other hand, if $C^{\prime}$ is of the same size as $C_{A}$. Then $C_{A}$ is a minimum vertex cover of $G[A, B]$, and so $x \notin C_{A}$. This means $C_{A} \cap N(x) \cap A \subseteq C_{A} \cap A$. And as $C_{A}$ is a minimum vertex cover of $G[A, B]$, we know from Lemma 2 that $C_{A} \cap N(x) \cap A \subseteq C^{\prime}$. In particular, this means $C^{\prime}$ covers all the edges of $G\left[A, B^{\prime}\right]$ not in $G[A, B]$, which means that $C^{\prime}$ is also a minimum vertex cover of $G\left[A, B^{\prime}\right]$. This latter observation means that $C^{\prime} \cap A \subseteq C_{A} \cap A$ from Lemma 2 .

$B \cap C_{A} \subseteq B \cap C^{\prime}$ : Suppose $C^{\prime}$ is smaller than $C_{A}$. This means $C^{\prime}+x$ is a minimum vertex cover of $G\left[A, B^{\prime}\right]$, and thus $B^{\prime} \cap\left(C^{\prime}+x\right) \supseteq B^{\prime} \cap C_{A}$. Which implies that $B \cap C^{\prime} \supseteq B \cap C_{A}$. Now assume that $C^{\prime}$ is of the same size as $C_{A}$. This means $C_{A}$ is a minimum vertex cover of $G[A, B]$ and $x \notin C_{A}$. Furthermore, this means $N(x) \cap A \subseteq C_{A} \cap A \subseteq C^{\prime} \cap A$ by Lemma 2 and we conclude that $C^{\prime}$ is a minimum vertex cover of $G\left[A, B^{\prime}\right]$. By Lemma 2, this means $B^{\prime} \cap C_{A} \subseteq B^{\prime} \cap C^{\prime}$ and in particular $B \cap C_{A} \subseteq B \cap C^{\prime}$.

$B \cap C^{\prime} \supseteq B \cap C$ : Suppose $C^{\prime}$ is smaller than $C$. This means $C^{\prime}+x$ is a minimum vertex cover of $G\left[A, B^{\prime}\right]$, and hence by Lemma 2 we have $B^{\prime} \cap\left(C^{\prime}+x\right) \subseteq B^{\prime} \cap C_{2}$, which implies $B \cap C^{\prime} \subseteq B \cap C_{2}$. Now suppose $C^{\prime}$ is of the same size as $C$. This means that $C$ is a minimum vertex cover of $G[A, B]$, and hence we immediately get $C \cap B \supseteq C^{\prime} \cap B$ by Lemma 2 .

This completes the proof, as we by transitivity of the subset relation have that $C_{A} \cap B \subseteq$ $C \cap B$, and $C \cap A \subseteq C_{A} \cap A$.

We are now ready to prove an important connectedness property of König covers that arise from cuts of a given branch decomposition.

- Lemma 4. Given a connected graph $G$ and rooted branch decomposition $(T, \delta)$ over $V(G)$, for any node $v$ in $T$, where $\mathcal{C}$ are the descendants of $v$ and $C_{u}$ means the $\delta(u)$-König cover of $G[\overline{\delta(u)}, \delta(u)]$, we have that

$$
\left(\bigcup_{x \in V(T) \backslash \mathcal{C}} C_{x}\right) \cap\left(\bigcup_{x \in \mathcal{C}} C_{x}\right) \subseteq C_{v} .
$$

Proof. For all $x \in \mathcal{C}$, since $C_{x}$ is a $\delta(x)$-König cover and $C_{v}$ is a minimum vertex cover, from Lemma 3, we have that $C_{x} \cap \overline{\delta(x)} \subseteq C_{v} \cap \overline{\delta(x)}$. In particular, since $\delta(x) \subseteq \delta(v)$, we 
have that $C_{x} \backslash \delta(v) \subseteq C_{v} \backslash \delta(v) \subseteq C_{v}$. Since each vertex of $V(G)$ is either in $\delta(v)$ or not in $\delta(v)$, by showing that also for all $x \in(V(T) \backslash \mathcal{C})$ we have $C_{x} \cap \delta(v) \subseteq C_{v}$ we can conclude that the lemma holds: For all $x \in V(T) \backslash \mathcal{C}$ either $\delta(v) \subseteq \delta(x)$ (when $x$ is an ancestor of $v$ ) or $\delta(v) \subseteq \overline{\delta(x)}$ (when $x$ is neither a descendant of $v$ nor an ancestor of $v$ ), in either case, we can apply the $\delta(v)$-König cover $C_{v}$ of $G[\delta(v), \overline{\delta(v)}]$ and the minimum vertex cover $C_{x}$ of $G[\delta(x), \overline{\delta(x)}]$ to Lemma 3 and see that $C_{x} \cap \delta(v) \subseteq C_{v} \cap \delta(v) \subseteq C_{v}$.

\subsection{The new characterization of $\mathrm{mmw}$}

We say a graph is nontrivial if it has an edge.

- Theorem 5. A nontrivial graph $G=(V, E)$ has $\operatorname{mmw}(G) \leq k$ if and only if there exist a tree $T$ of max degree at most 3 and for each vertex $u \in V$ a nontrivial subtree $T_{u}$ of $T$ such that (i) if $u v \in E$ then the subtrees $T_{u}$ and $T_{v}$ have at least one vertex of $T$ in common, and (ii) for every edge of $T$ there are at most $k$ subtrees using this edge.

Proof. Forward direction: Let $(T, \delta)$ be a rooted branch decomposition over $V$ having mmwidth at most $k$, and assume $G$ has no isolated vertices. For each edge $e=u v$ of $T$, with $u$ a child of $v$, assign the $\delta(u)$-König cover $C_{u}$ of $G[\delta(u), V \backslash \delta(u)]$ to the edge $u v$. For each vertex $x$ of $G$, define the set of edges of $T$ whose König cover contains $x$ and let $T_{x}$ be the sub-forest of $T$ induced by these edges. Using Lemma 4 we first show that $T_{x}$ is a connected forest and thus a subtree of $T$. Consider two vertices $u$ and $v$ such that $x \in C_{u} \cap C_{v}$. Let $p$ be the lowest common ancestor of $u$ and $v$. For every vertex $w$ on the path from $p$ to $u$ and on the path from $p$ to $v$, except $p$, we know that exactly one of $u, v$ is a descendant of $w$. By Lemma $4,\left(C_{u} \cap C_{v}\right) \subseteq C_{w}$. It means that if a vertex $x$ of $G$ is in both $C_{u}$ and $C_{v}$ then it is also in $C_{w}$, which implies that $T_{x}$ is connected.

Now, since the branch decomposition has mm-width at most $k$ part (ii) in the statement of the Theorem holds. For an arbitrary edge $a b$ of $G$, consider any edge $e$ of $T$ on the path from $\delta^{-1}(a)$ to $\delta^{-1}(b)$ and the partition $(A, B)$ induced by $e$ where $a \in A, b \in B$. Then the König cover of $e$ must contain one of $a$ and $b$, and thus, (i) holds as well. Finally, $T_{x}$ is nontrivial because the edge of $T$ incident with a leaf $\delta^{-1}(x)$ assigns the König cover $\{x\}$. If $G$ has isolated isolated vertices, $T_{x}$ is not nontrivial for isolated vertex $x$. We fix this by setting $T_{x}$ to consist exactly of the edge incident with $\delta^{-1}(x)$, for any isolated vertex $x$ of $G$.

Backward direction: For each given subtree $\left\{T_{u}\right\}_{u \in V}$ of $T$, choose an edge in $T_{u}$ (it is also in $T$ ) and append in the tree $T$ a leaf $\ell_{u}$, and extend $T_{u}$ to contain $\ell_{u}$ and set $\delta\left(\ell_{u}\right)=u$. Exhaustively remove leaves (from both $T$ and the subtrees) that are not mapped by $\delta$. Call the resulting tree $T^{\prime}$ and subtrees $\left\{T_{u}^{\prime}\right\}_{u \in V}$. Note that subtrees $\left\{T_{u}^{\prime}\right\}_{u \in V}$ and $T^{\prime}$ still satisfy (i) and (ii). We claim that $\left(T^{\prime}, \delta\right)$ is a branch decomposition of mm-width at most $k$. It is clearly a branch decomposition over $V$, and for any edge $e$ of $T^{\prime}$, if we choose $S \subseteq V$ to be those $u$ with $T_{u}$ using this edge $e$, then this will be a vertex cover of the bipartite graph $H$ given by this edge $e$, and of size at most $k$ because for an edge $x y$ in $H$, one of $T_{x}$ and $T_{y}$ must contain $e$.

In the Introduction we mentioned analogous characterizations of treewidth and branchwidth, for these see e.g. [14]. Another alternative characterization is the following.

Corollary 6. A graph $G$ has $\operatorname{mmw}(G) \leq k$ if and only if it is a subgraph of a chordal graph $H$ and for every maximal clique $X$ of $H$ there exists $A, B, C \subseteq X$ with $A \cup B \cup C=X$ and $|A|,|B|,|C| \leq k$ such that any subset of $X$ that is a minimal separator of $H$ is a subset of either $A, B$ or $C$. 
We only sketch the proof, which is similar to an alternative characterization of branchwidth given in [14]. We say a tree is ternary if it has maximum degree at most 3. Note that a graph is chordal if and only if it is an intersection graph of subtrees of a tree [8]. In the forward direction, take the chordal graph resulting from the subtrees of ternary tree representation. In the backward direction, take a clique tree of $H$ and make a ternary tree decomposition (which is easily made into a subtrees of ternary tree representation) by for each maximal clique $X$ of degree larger than three making a bag $X$ with three neighboring bags $A, B, C$. If minimal separators $S_{1}, \ldots, S_{q} \subseteq X$ are contained in $A$ make a path extending from bag $A$ of $q$ new bags also containing $A$, with a single bag containing $S_{i}, 1 \leq i \leq q$, attached to each of them. These ternary subtrees, one for each maximal clique, is then connected together in a tree by the structure of the clique tree, adding an edge between bags of identical minimal separators.

\section{Fast DP for Dominating Set parameterized by mm-width}

For graph $G=(V, E)$ a subset of vertices $S \subseteq V$ is said to dominate the vertices in $N[S]$, and it is a dominating set if $N[S]=V$. Given a rooted branch decomposition $(T, \delta)$ of $G$ of mm-width $k$, we will in this section give an $O^{*}\left(8^{k}\right)$ algorithm for computing the size of a Minimum Dominating Set of $G$. This is an algorithm doing dynamic programming along a rooted tree decomposition $\left(T^{\prime},\left\{X_{t}\right\}_{t \in V\left(T^{\prime}\right)}\right)$ of $G$ that we compute from $(T, \delta)$ as follows.

Given a rooted branch decomposition $(T, \delta)$ of $G$ having mm-width $k$ the proof of Theorem 5 yields a polynomial-time algorithm (using an algorithm for maximum matching in bipartite graphs) finding a family $\left\{T_{u}\right\}_{u \in V(G)}$ of nontrivial subtrees of $T$ (note we can assume $T$ is a rooted tree with root of degree two and all other internal vertices of degree three) such that (i) if $u v \in E(G)$ then the subtrees $T_{u}$ and $T_{v}$ have at least one vertex of $T$ in common, and (ii) for every edge of $T$ there are at most $k$ subtrees using this edge. From this it is easy to construct a rooted tree decomposition $\left(T^{\prime},\left\{X_{t}\right\}_{t \in V\left(T^{\prime}\right)}\right)$ of $G$, having the properties described in Figure 2. Let $T^{\prime}$ be a tree with vertex set $A \cup B \cup\{r\}$ where $A$ is the set of edges of $T, B$ is the set of non-root vertices (all of degree-3) of $T$, and $r$ is the root of $T$ and also the root of $T^{\prime}$. Two vertices $e, v$ of $T^{\prime}$ are adjacent if and only if $e \in A$ and $v \in B \cup\{r\}$ are incident in $T$. For a vertex $e \in A$, let $X_{e}$ be the set of vertices in $G$ such that if a subtree $T_{w}$ uses edge $e$ of $T$, then $w \in X_{e}$. For a vertex $v \in B$, let $X_{v}$ be the set of vertices in $G$ such that for the three incident edges $e_{1}, e_{2}, e_{3}$ of $v$ in $T, X_{v}=X_{e_{1}} \cup X_{e_{2}} \cup X_{e_{3}}$. Let $X_{r}=X_{e_{1}} \cup X_{e_{2}}$ if $e_{1}$ and $e_{2}$ are incident with $r$ in $T$. Then $\left(T^{\prime},\left\{X_{t}\right\}_{t \in V\left(T^{\prime}\right)}\right)$ is a tree decomposition of $G$ with a root $r$, having the properties described in Figure 2, which we will use in the dynamic programming.

Let us now define the relevant subproblems for the dynamic programming over this tree decomposition. For node $t$ of the tree we denote by $G_{t}$ the graph induced by the union of $X_{u}$ where $u$ is a descendant of $t$. A coloring of a bag $X_{t}$ is a mapping $f: X_{t} \rightarrow\{1,0, *\}$ with the meaning that: all vertices with color 1 are contained in the dominating set of this partial solution in $G_{t}$, all vertices with color 0 are dominated, while vertices with color * might be dominated, not dominated, or in the dominating set. Thus the only restriction is that a vertex with color 1 must be a dominator, and a vertex with color 0 must be dominated. Thus, for any $S \subseteq V(G)$ there is a set $c(S)$ of $3^{|S|} 2^{|N(S)|}$ colorings $f: V(G) \rightarrow\{1,0, *\}$ compatible with taking $S$ as set of dominators, with vertices of $S$ colored 1, 0 or $*$, vertices of $N(S)$ colored 0 or $*$, and the remaining vertices colored $*$.

For a coloring $f$ of bag $X_{t}$ we denote by $T[t, f]$ (and view this as a 'Table' of values) the minimum $|S|$ over all $S \subseteq V\left(G_{t}\right)$ such that there exists $f^{\prime} \in c(S)$ with $\left.f^{\prime}\right|_{X_{t}}=f$ and 

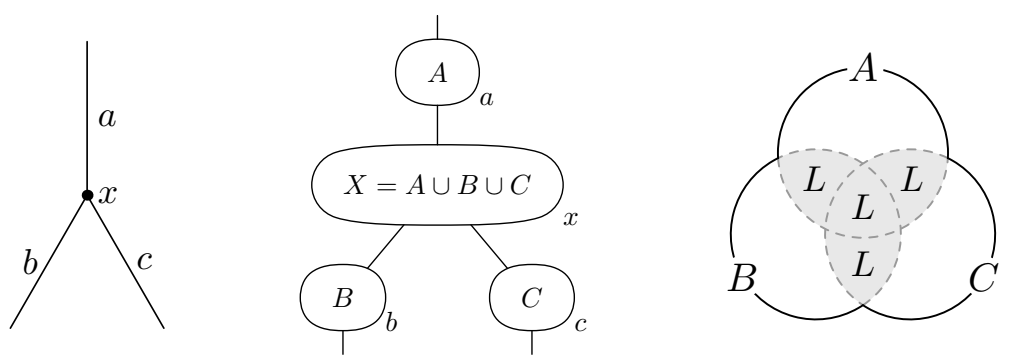

Figure 2 Part of ternary tree used in the subtree representation of $G$ on the left, with node $x$ having three incident edges $a, b, c$, with subtrees of vertices contained in $A, B, C \subseteq V(G)$ using these edges respectively, giving rise to the four bags in the tree decomposition shown in the middle, with constraint $|A|,|B|,|C| \leq k$.

$\left.f^{\prime}\right|_{V\left(G_{t}\right) \backslash X_{t}}$ having everywhere the value 0 . In other words, the minimum size of a set $S$ of vertices of $G_{t}$ that dominate all vertices in $V\left(G_{t}\right) \backslash X_{t}$, with a coloring $f^{\prime}$ compatible with taking $S$ as set of dominators, such that $f^{\prime}$ restricted to $X_{t}$ gives $f$. If no such set $S$ exists, then $T[t, f]=\infty$. Note that the size of the minimum dominating set of $G$ is the minimum value over all $T[r, f]$ where $f^{-1}(*)=\emptyset$ at the root $r$. We initialize the table at a leaf $\ell$, with $X_{\ell}=\{v\}$ as follows. Denote by $f_{i}$ the coloring from $\{v\}$ to $\{1,0, *\}$ with $f_{i}(v)=i$ for $i \in\{1,0, *\}$. Then for a leaf bag $X_{\ell}$, set $T\left[\ell, f_{1}\right]:=1, T\left[\ell, f_{0}\right]:=\infty, T\left[\ell, f_{*}\right]:=0$.

For internal nodes of the tree, instead of separate 'Join, Introduce and Forget' operations we will give a single update rule with several stages. We will be using an Extend-Table subroutine which takes a partially filled table $T[t, \cdot]$ and extends it to table $T^{\prime}[t, \cdot]$ so the result will adhere to the above definition, ensuring the monotonicity property that $T^{\prime}[t, f] \leq T^{\prime}\left[t, f^{\prime}\right]$ for any $f$ we can get from $f^{\prime}$ by changing the color of a vertex from 1 to 0 or $*$, or from 0 to *. Extend-Table is implemented as follows:

(a) Initialize. For all $f$, if $T[t, f]$ is defined then $T^{\prime}[t, f]:=T[t, f]$, else $T^{\prime}[t, f]:=\infty$.

(b) Change from 1 to 0 . For $q=\left|X_{t}\right|$ down to 1 : for any $f$ in $T^{\prime}[t, f]$ where $\mid\{v: f(v)=$ $1\} \mid=q$, for any choice of a single vertex $u \in\{v: f(v)=1\}$ set $f_{u}(u)=0$ and set $f_{u}(x)=f(x)$ for $x \neq u$, and update $T^{\prime}\left[t, f_{u}\right]:=\min \left\{T^{\prime}\left[t, f_{u}\right], T^{\prime}[t, f]\right\}$.

(c) Change from 0 to $*$. Similarly as in step (b).

Note the transition from color 1 to $*$ will happen by transitivity. The time for Extend-Table is proportional to the number of entries in the tables times $\left|X_{t}\right|$.

Assume we have the situation in Figure 2, corresponding to the bags surrounding any degree-three node $x$ of the tree decomposition. This arises from the branch decomposition (and the subtrees of tree representation) having a node incident to three edges, creating three bags $a, b, c$ containing subsets of vertices $A, B, C$, respectively, each of size at most $k$, and giving rise to the four bags $a, b, c, x$ in Figure 2, with the latter containing subsets of vertices $X=A \cup B \cup C$. Let $L=(A \cap B) \cup(A \cap C) \cup(B \cap C)$. Assume we have already computed $T[b, f]$ and $T[c, f]$ for all $3^{|B|}$ and $3^{|C|}$ choices of $f$, respectively. We want to compute $T[a, f]$ for all $3^{|A|}$ choices of $f$, in time $O^{*}\left(\max \left\{3^{|A|}, 3^{|B|}, 3^{|C|}, 3^{|L|} 2^{|X \backslash L|}\right\}\right)$. Note that we will not compute the table $T[x, \cdot]$, as it would have $3^{|X|}$ entries, which is more than the allowed time bound. Instead, we compute a series of tables:

(1) $T_{b}^{1}[x, \cdot]$ (and $\left.T_{c}^{1}[x, \cdot]\right)$ of size $3^{|B|}$, by for each entry $T[b, f]$ extending the coloring $f$ of $B$ to a unique coloring $f^{\prime}$ of $X$ based on the neighborhood of the dominators in $f$

(2) $T_{b}^{2}[x, \cdot]$ (and $T_{c}^{2}[x, \cdot]$ ) of size at most $\min \left(3^{|B|}, 3^{|B \cap L|} 2^{|X \backslash(B \cap L)|}\right.$ ), by changing each coloring $f$ of $X$ to a coloring $f^{\prime}$ of $X$ where vertices in $B \backslash L$ having color 1 instead are given color 0 (note these vertices have no neighbors in $V(G) \backslash V\left(G_{x}\right)$ ) 
(3) $T_{b}^{3}[x, \cdot]$ (and $\left.T_{c}^{3}[x, \cdot]\right)$ of size exactly $3^{|B \cap L|} 2^{|X \backslash(B \cap L)|}$, with $f^{-1}(1) \subseteq B \cap L$, by running Extend-Table on $T_{b}^{2}[x, \cdot]$

(4) $T_{s c}^{1}[x, \cdot]$ of size $3^{|L|} 2^{|X \backslash L|}$ by subset convolution over parts of $T_{b}^{3}[x, \cdot]$ and $T_{c}^{3}[x, \cdot]$

(5) $T_{s c}^{2}[x, \cdot]$ of size $3^{|L|} 2^{|X \backslash L|}$ by running Extend-Table on $T_{s c}^{1}[x, \cdot]$

(6) $T[a, \cdot]$ of size $3^{|A|}$ by going over all $3^{|A|}$ colorings of $A$ and minimizing over appropriate entries of $T_{s c}^{2}[x, \cdot]$

Note that in step 4 we use the following:

- Theorem 7 (Fast Subset Convolution [2]). For two functions $g, h: 2^{V} \rightarrow\{-M, \ldots, M\}$, given all the $2^{|V|}$ values of $g$ and $h$ in the input, all $2^{|V|}$ values of the subset convolution of $g$ and $h$ over the integer min-sum semiring, i.e. $(g * h)(Y)=\min _{Q \cup R=Y \text { and } Q \cap R=\emptyset} g(Q)+h(R)$, can be computed in time $2^{|V|}|V|^{O(1)} \cdot O(M \log M \log \log M)$.

Let us now give the details of the first three steps:

(1) Compute $T_{b}^{1}[x, \cdot]$. In any order, go through all $f: B \rightarrow\{1,0, *\}$ and compute $f^{\prime}$ : $B \cup A \cup C \rightarrow\{1,0, *\}$ by

$$
f^{\prime}(v)= \begin{cases}f(x) & \text { if } v \in B \\ 0 & \text { if } v \notin B \text { and } \exists u \in B: f(u)=1 \wedge u v \in E(G) \\ * & \text { otherwise }\end{cases}
$$

and set $T_{b}^{1}\left[x, f^{\prime}\right]:=T[b, f]$.

(2) Compute $T_{b}^{2}[x, \cdot]$. First, initialize $T_{b}^{2}[x, f]=\infty$ for all $f: B \cup A \cup C \rightarrow\{1,0, *\}$ where $f^{-1}(1) \subseteq B \cap L$. In any order, go through all $f: B \cup A \cup C \rightarrow\{1,0, *\}$ such that $T_{b}^{1}[x, f]$ was defined in the previous step, and compute $f^{\prime}: B \cup A \cup C \rightarrow\{1,0, *\}$ by

$$
f^{\prime}(v)= \begin{cases}0 & \text { if } v \in B \backslash L \text { and } f(v)=1 \\ f^{\prime}(v)=f(v) & \text { otherwise }\end{cases}
$$

and set $T_{b}^{2}\left[x, f^{\prime}\right]:=\min \left\{T_{b}^{2}\left[x, f^{\prime}\right], T_{b}^{1}[x, f]\right\}$. There will be no other entries in $T_{b}^{2}[x, \cdot]$.

(3) Compute $T_{b}^{3}[x, \cdot]$ by Extend-Table on $T_{b}^{2}[x, \cdot]$.

The total time for the above three steps is bounded by $O^{*}\left(\max \left\{3^{|B|}, 3^{|B \cap L|} 2^{|X \backslash(B \cap L)|}\right\}\right)$. Note that $T_{b}^{3}[x, f]$ is defined for all $f$ where vertices in $B \cap L$ take on values $\{1,0, *\}$ and vertices in $X \backslash(B \cap L)$ take on values $\{0, *\}$. The value of $T_{b}^{3}[x, f]$ will be the minimum $|S|$ over all $S \subseteq V\left(G_{b}\right)$ such that there exists $f^{\prime} \in c(S)$ with $\left.f^{\prime}\right|_{X}=f$ and $\left.f^{\prime}\right|_{V\left(G_{b}\right) \backslash X}$ having everywhere the value 0 . Note the slight difference from the standard definition, namely that even though the coloring $f$ is defined on $X$, the dominators only come from $V\left(G_{b}\right)$, and not from $V\left(G_{x}\right)$. The table $T_{c}^{3}[x, \cdot]$ is computed in a similar way, with the colorings again defined on $X$ but with the dominators now coming from $V\left(G_{c}\right)$.

When computing a Join of these two tables, we want dominators to come from $V\left(G_{b}\right) \cup$ $V\left(G_{c}\right)$. Because of the monotonicity property that holds for these two tables, we can compute their Join $T_{s c}^{1}[x, f]$ for any $f$ where vertices in $L$ take on values $\{1,0, *\}$ and vertices in $X \backslash L$ take on values $\{0, *\}$, by combining colorings as follows:

$$
T_{s c}^{1}[x, f]=\min _{f_{b}, f_{c}}\left(T_{b}^{3}\left[x, f_{b}\right]+T_{c}^{3}\left[x, f_{c}\right]\right)-\left|f^{-1}(1) \cap B \cap C\right|
$$

where $f_{b}, f_{c}$ satisfy:

- $f(v)=0$ if and only if $\left(f_{b}(v), f_{c}(v)\right) \in\{(0, *),(*, 0)\}$

- $f(v)=*$ if and only if $f_{b}(v)=f_{c}(v)=*$

- $f(v)=1$ if and only if $v \in B \cap C$ and $f_{b}(v)=f_{c}(v)=1$, or $v \in B \backslash C$ and $\left(f_{b}(v), f_{c}(v)\right)=$ $(1, *)$, or $v \in C \backslash B$ and $\left(f_{b}(v), f_{c}(v)\right)=(*, 1)$. 
This means that we can apply subset convolution to compute a table $T_{s c}^{1}[x, f]$ on $3^{|L|} 2^{|X \backslash L|}$ entries based on $T_{b}^{3}[x, f]$ and $T_{c}^{3}[x, f]$. Note that $(B \cap L) \cup(C \cap L)=L$. For this step we follow the description in [6, Section 11.1.2]. Fix a set $D \subseteq L$ to be the dominating vertices. Let $F_{D}$ denote the set of $2^{|X \backslash D|}$ functions $f: X \rightarrow\{1,0, *\}$ such that $f^{-1}(1)=D$, i.e. with vertices in $X \backslash D$ mapping in all possible ways to $\{0, *\}$. For each $D \subseteq L$ we will by subset convolution compute the values of $T_{s c}^{1}[x, f]$ for all $f \in F_{D}$.

We represent every $f \in F_{D}$ by the set $S=f^{-1}(0)$ and define $b_{S}: X \rightarrow\{1,0, *\}$ such that $b_{S}(x)=1$ if $x \in D \cap B, b_{S}(x)=0$ if $x \in S, b_{S}(x)=*$ otherwise. Similarly, define $c_{S}: X \rightarrow\{1,0, *\}$ such that $c_{S}(x)=1$ if $x \in D \cap C, c_{S}(x)=0$ if $x \in S, c_{S}(x)=*$ otherwise. Then, as explained previously, for every $f \in F_{D}$ we want to compute

$$
T_{s c}^{1}[x, f]=\min _{Q \cup R=f^{-1}(0) \text { and } Q \cap R=\emptyset}\left(T_{b}^{3}\left[x, b_{Q}\right]+T_{c}^{3}\left[x, c_{R}\right]\right)-\left|f^{-1}(1) \cap B \cap C\right| .
$$

Define functions $T_{b}: 2^{X \backslash D} \rightarrow \mathbb{N}$ such that for every $S \subseteq X \backslash D$ we have $T_{b}(S)=T_{b}^{3}\left[x, b_{S}\right]$. Likewise, define functions $T_{c}: 2^{X \backslash D} \rightarrow \mathbb{N}$ such that for every $S \subseteq X \backslash D$ we have $T_{c}(S)=$ $T_{c}^{3}\left[x, c_{S}\right]$. Also, define $a_{S}: X \rightarrow\{1,0, *\}$ such that $a_{S}(x)=1$ if $x \in D, a_{S}(x)=0$ if $x \in S$, $a_{S}(x)=*$ otherwise. We then compute for every $S \subseteq X \backslash D$,

$$
T_{s c}^{1}\left[x, a_{S}\right]:=\left(T_{b} * T_{c}\right)(S)-\left|f^{-1}(1) \cap B \cap C\right|
$$

where the subset convolution is over the mini-sum semiring.

(4) In step (4), by Fast Subset Convolution, Theorem 7, we compute $T_{s c}^{1}\left[x, a_{S}\right]$, for all $a_{S}$ defined by all $f \in F_{D}$, in $O^{*}\left(2^{|X \backslash D|}\right)$ time each. For all such subsets $D \subseteq L$ we get the time

$$
\sum_{D \subseteq L} 2^{|X \backslash D|}=\sum_{D \subseteq L} 2^{|X \backslash L|} 2^{|L \backslash D|}=2^{|X \backslash L|} \sum_{D \subseteq L} 2^{|L \backslash D|}=2^{|X \backslash L|} 3^{|L|} .
$$

(5) In step (5) we need to run Extend-Table on $T_{s c}^{1}[x, \cdot]$ to get the table $T_{s c}^{2}[x, \cdot]$. This since the subset convolution was computed for each fixed set of dominators so the monotonicity property of the table may not hold. Note that the value of $T_{s c}^{2}[x, f]$ will be the minimum $|S|$ over all $S \subseteq V\left(G_{b}\right) \cup V\left(G_{c}\right)$ such that there exists $f^{\prime} \in c(S)$ with $\left.f^{\prime}\right|_{X}=f$ and $\left.f^{\prime}\right|_{\left(V\left(G_{b}\right) \cup V\left(G_{c}\right)\right) \backslash X}$ having everywhere the value 0 .

(6) In step (6) we will for each $f: A \rightarrow\{1,0, *\}$ compute $f^{\prime}: B \cup A \cup C \rightarrow\{1,0, *\}$ by

$$
f^{\prime}(v)= \begin{cases}1 & \text { if } v \in A \cap L \text { and } f(v)=1 \\ 0 & \text { if } v \in A \text { and } f(v)=0 \text { and } N(v) \cap f^{-1}(1)=\emptyset \\ 0 & \text { if } v \notin A \text { and } N(v) \cap f^{-1}(1)=\emptyset \\ * & \text { otherwise }\end{cases}
$$

and set $T[a, f]:=T_{s c}^{2}\left[x, f^{\prime}\right]+\left|f^{-1}(1) \cap(A \backslash L)\right|$.

Note that when we iterate over all choices of $f: A \rightarrow\{1,0, *\}$, the vertices colored 0 (in addition to all vertices of $X \backslash A)$ must either be dominated by the vertices in $f^{-1}(1)$ or by vertices in $X \backslash V_{a}$. As we know precisely what vertices of $f^{-1}(0)$ are dominated by $f^{-1}(1)$, we know the rest must be dominated from vertices of $X \backslash V_{a}$, and therefore we look in $T_{s c}\left[x, f^{\prime}\right]$ at an index $f^{\prime}$ which colors the rest of $f^{-1}(0)$ by 0 . We can also observe that it is not important for us whether or not $f^{-1}(0)$ contains all neighbours of $f^{-1}(1)$, since we are iterating over all choices of $f$ - also those where $f^{-1}(0)$ contains all neighbours of $f^{-1}(1)$.

The total runtime becomes $O^{*}\left(\max \left\{3^{|A|}, 3^{|B|}, 3^{|C|}, 3^{|L|} 2^{|(A \cup B \cup C) \backslash L|}\right\}\right)$, with $L=(A \cap$ $B) \cup(A \cap C) \cup(B \cap C)$ and with constraints $|A|,|B|,|C| \leq k$. This runtime is maximum when $L=\emptyset$, giving a runtime of $O^{*}\left(2^{3 k}\right)$. We thus have the following theorem.

Theorem 8. Given a graph $G$ and branch decomposition over its vertex set of mm-width $k$ we can solve Dominating Set in time $O^{*}\left(8^{k}\right)$. 


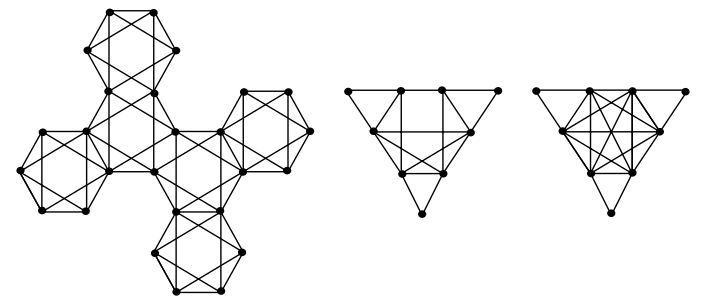

Figure 3 Three graphs of mm-width 2. Left, middle have treewidth 4, and right has treewidth 5 .

\section{Discussion}

We have shown that the graph parameter mm-width will for some graphs be better than treewidth for solving Minimum Dominating Set. The improvement holds whenever $\operatorname{tw}(G)>$ $1.549 \times \operatorname{mmw}(G)$, if given only the graph as input. In Figure 3 we list some examples of small graphs having treewidth at least twice as big as mm-width. It could be interesting to explore the relation between treewidth and mm-width for various well-known classes of graphs. The given algorithmic technique, using fast subset convolution, should extend to any graph problem expressible as a maximization or minimization over $(\sigma, \rho)$-sets, using the techniques introduced for treewidth in [17].

We may also compare with branchwidth. Let $\omega$ be the exponent of matrix multiplication, which is less than 2.3728639 [11]. In 2010, Bodlaender, van Leeuwen, van Rooij, and Vatshelle [5] gave an $O^{*}\left(3^{\frac{\omega}{2} k}\right)$ time algorithm solving Minimum Dominating Set if an input graph is given with its branch decomposition of width $k$. This means that given decompositions of $\operatorname{bw}(G)$ and $\operatorname{mmw}(G)$ our algorithm based on mm-width is faster than the algorithm in [5] whenever bw $(G)>\log _{3} 8 \cdot \frac{2}{\omega} \cdot \operatorname{mmw}(G)>\frac{2 \log _{3} 8}{2.3728639} \cdot \operatorname{mmw}(G)>1.6 \mathrm{mmw}(G)$.

Taking the subtrees of tree representation for treewidth, branchwidth and maximum matching width mentioned in the Introduction as input, our algorithm for dominating set can be seen as a generic one that works for any of treewidth, branchwidth or maximum matching width of the given representation, and in case of both treewidth and mm-width it will give the best runtime known.

We gave an alternative definition of mm-width using subtrees of a tree, similar to alternative definitions of treewidth and branchwidth. We saw that in the subtrees of a tree representation treewidth focuses on nodes, branchwidth focuses on edges, and mm-width combines them both. There is also a fourth way of defining a parameter through these intersections of subtrees representation; where subtrees $T_{u}$ and $T_{v}$ must share an edge if $u v \in E(G)$ (similar to branchwidth) and the width is defined by the maximum number of subtrees sharing a single vertex (similar to treewidth). This parameter will be an upper bound on all the other three parameters, but might it be that the structure this parameter highlights can be used to improve the runtime of Dominating Set beyond $O^{*}\left(3^{\text {tw }(G)}\right)$ for even more cases than those shown using mm-width and branchwidth?

\section{References}

1 Eyal Amir. Approximation algorithms for treewidth. Algorithmica, 56(4):448-479, 2010.

2 Andreas Björklund, Thore Husfeldt, Petteri Kaski, and Mikko Koivisto. Fourier meets Möbius: fast subset convolution. In STOC'O7 - Proceedings of the 39th Annual ACM Symposium on Theory of Computing, pages 67-74. ACM, New York, 2007.

3 Hans L Bodlaender, Pal Gronas Drange, Markus S Dregi, Fedor V Fomin, Daniel Lokshtanov, and Michal Pilipczuk. An $o\left(c^{k} n\right)$ 5-approximation algorithm for treewidth. In 
Foundations of Computer Science (FOCS), 2013 IEEE 54th Annual Symposium on, pages 499-508. IEEE, 2013.

4 Hans L. Bodlaender and Ton Kloks. Efficient and constructive algorithms for the pathwidth and treewidth of graphs. J. Algorithms, 21(2):358-402, 1996.

5 Hans L. Bodlaender, Erik Jan van Leeuwen, Johan M. M. van Rooij, and Martin Vatshelle. Faster algorithms on branch and clique decompositions. In Mathematical foundations of computer science 2010, volume 6281 of Lecture Notes in Comput. Sci., pages 174-185. Springer, Berlin, 2010.

6 Marek Cygan, Fedor V. Fomin, Łukasz Kowalik, Daniel Lokshtanov, Dániel Marx, Marcin Pilipczuk, Michał Pilipczuk, and Saket Saurabh. Parameterized Algorithms. Springer International Publishing, New York, 2016.

7 Reinhard Diestel. Graph theory, volume 173 of Graduate Texts in Mathematics. Springer, Heidelberg, fourth edition, 2010.

8 Fănică Gavril. The intersection graphs of subtrees in trees are exactly the chordal graphs. J. Combinatorial Theory Ser. B, 16:47-56, 1974.

9 Jisu Jeong, Sigve Hortemo Sæther, and Jan Arne Telle. An FPT algorithm computing a decomposition of optimal mm-width. in preparation, 2015.

10 Dénes König. Gráfok és mátrixok. Matematikai és Fizikai Lapok, 38:116-119, 1931.

11 François Le Gall. Powers of tensors and fast matrix multiplication. In ISSAC 2014 Proceedings of the 39th International Symposium on Symbolic and Algebraic Computation, pages 296-303. ACM, New York, 2014.

12 Daniel Lokshtanov, Dániel Marx, and Saket Saurabh. Known algorithms on graphs of bounded treewidth are probably optimal. In Proceedings of the Twenty-Second Annual ACM-SIAM Symposium on Discrete Algorithms, pages 777-789. SIAM, Philadelphia, PA, 2011.

13 Sang-il Oum and Paul Seymour. Approximating clique-width and branch-width. J. Combin. Theory Ser. B, 96(4):514-528, 2006.

14 Christophe Paul and Jan Arne Telle. Edge-maximal graphs of branchwidth $k$ : the $k$ branches. Discrete Math., 309(6):1467-1475, 2009.

15 Neil Robertson and P. D. Seymour. Graph minors. X. Obstructions to tree-decomposition. J. Combin. Theory Ser. B, 52(2):153-190, 1991.

16 Sigve Hortemo Sæther and Jan Arne Telle. Between treewidth and clique-width. In Graphtheoretic concepts in computer science, volume 8747 of Lecture Notes in Comput. Sci., pages 396-407. Springer, Cham, 2014.

17 Johan M. M. van Rooij, Hans L. Bodlaender, and Peter Rossmanith. Dynamic programming on tree decompositions using generalised fast subset convolution. In Algorithms - ESA 2009, volume 5757 of Lecture Notes in Comput. Sci., pages 566-577. Springer, Berlin, 2009.

18 Martin Vatshelle. New Width Parameters of Graphs. PhD thesis, University of Bergen, 2012. 